\title{
Halo Orbits at Sun-Mars L1, L2 in the Photogravitational Restricted Three-Body Problem with Oblateness
}

\author{
Nishanth Pushparaj ${ }^{1}$, Ram Krishan Sharma ${ }^{2}$ \\ ${ }^{1}$ Junior Reserach Fellow, Department of Aerospace Engineering, Karunya University, Coimbatore, India \\ ${ }^{2}$ Professor, Department of Aerospace Engineering, Karunya University, Coimbatore, India \\ Email: ${ }^{1}$ p.nishanth12@gmail.com ${ }^{2}$ ramkrishan@karunya.edu
}

\begin{abstract}
Photogravitational Restricted Three-Body Problem (PRTBP) with smaller primary being an oblate spheroid with its equatorial plane coincident with the plane of motion of the primaries is considered and halo orbits in the vicinity of Sun-Mars Lagrangian points L1 and L2 are computed numerically. The effects of perturbations on size, shape, location and time period of the halo orbits are studied. It is found that the increase in solar radiation pressure at constant oblateness elongates the halo orbits at L1 and the orbits move towards the radiating body. At L2, the halo orbits shrink and move towards the smaller primary with increase in solar radiation pressure at constant oblateness. For constant radiation pressure, increase in oblateness causes the location of L1 and L2 halo orbits to move away from the smaller primary. The time period of L1 halo orbits increases with increase in radiation pressure for constant oblateness and decreases with increase in oblateness for constant radiation pressure. However, the effect of solar radiation pressure and oblateness for L2 halo orbits is reversed.
\end{abstract}

Keywords: Photogravitational restricted three-body problem; Lagrangian points; Halo orbits; time period, oblateness, solar radiation pressure.

\section{Introduction}

Interplanetary missions to the Mars have become a topic of increasing debate over the last few years. There are considerable techniques to transfer a spacecraft or a space probe from an Earth Parking Orbit (EPO) to an orbit about the Mars, including direct transfers, low-thrust transfers, and low-energy ballistic transfers. The conventional direct method has been used by many missions, including Mariner program. Its greatest advantage when compared with other transfer techniques is its short transfer time; its greatest weakness is its high fuel cost. The low-thrust methods have an advantage of lower fuel cost and the disadvantages include the long transfer time and the complexity of mission designs. The lowenergy transfer technique generally balances the advantages and disadvantages of the former transfer techniques: it requires less fuel than a direct transfer and less transfer time than a modern low-thrust transfer technique. For the application of such low-energy transfer from EPO to an orbit about Mars, we apply dynamical systems theory to the construction of these transfers by requiring that the spacecraft's destination is final or an intermediate staging orbit near Mars. Interest in the liberation points for space missions has continued to increase and inspire even more challenging objectives that are reflected in some of the current missions such as SOHO, MAP and Genesis. Fundamental knowledge of orbital mechanics on launch, orbit design, orbital prediction, manoeuvres, station keeping and navigation are sufficient. However, the interplanetary missions become more complex and further understanding of astrodynamics is required.

The five Lagrange's liberation points are the equilibrium solutions of the Circular Restricted ThreeBody Problem (CRTBP). Szebehely [1] is an excellent treatise on restricted three-body problem, which includes detailed study on the Lagrangian points. Applications of liberation point orbits are intensely studied by Farquhar and Kamel [2] on quasi-periodic orbits about translunar liberation points. Mission designs utilizing the halo orbits become more challenging and several works have been done by Farquhar et al [3], Richardson [4], Huber et al[5], Go mez et al [6][7], Rausch [8], Nakamiya et al [9], Koon et al [10], Lu Jing et al [11] and Nath and Ramanan [12]. The subject of periodic solutions of the CRTBP has 
received enormous attention in the past few decades. Since the late twentieth century, enormous amount of research has enriched the study of CRTBP, but the influence of the various perturbing forces has not been studied in many of such interesting problems. The classical model does not account for some of the perturbing forces such as oblateness, solar radiation pressure, Poynting-Robertson drag effects and variations of the masses of the primaries. Some of the significant works in the PRTBP were done by Radzievskii [13], Chernikov [14], Bhatnagar and Chawla [15], Sharma [16], Kunitsyn and Tureshbaev [17] and Lukyanov [18].

Three dimensional halo orbits in CRTBP was studied by Breakwell and Brown [19], Howell [20][21] and Howell et al [22]. The PRTBP with oblateness effect arises from the classical problem if at least one of the primaries is an intense emitter of radiation and the other primary is an oblate spheroid as in Sharma [16] and Tiwary and Kushvah [23]. Radzievskii [13] formulated the PRTBP and studied it for three specific bodies: Sun, a planet and a dust particle. The radiation repulsive force exerted on the particle can be represented in terms of gravitational attraction $\mathrm{F}_{\mathrm{g}}[13]$ as:

$$
F=F_{g}-F_{p}=F_{g}\left(1-\frac{F_{p}}{F_{g}}\right)=q F_{g}, \quad F_{p}=F_{g}(1-q) \text {. }
$$

Here $q$, a constant for a given particle, is a mass reduction factor and is given in terms of particle radius $a$, density $(\delta)$ and radiation efficiency factor $x$ as:

$$
q=1-\frac{5.6 \times 10^{-3}}{a(\delta)} . x \quad \text { (c.g.s. units), } q=1-\varepsilon .
$$

The assumption that $q$ is constant implies that the fluctuations in the beam of solar radiation and the effect of planet's shadow are neglected. Typical values for interplanetary dust particles are in the range of 50 to $500 \mu \mathrm{m}$ and their densities range between $1 \mathrm{~g} / \mathrm{cm}^{3}$ and $3 \mathrm{~g} / \mathrm{cm}^{3}$ with an average density of $2 \mathrm{~g} / \mathrm{cm}^{3}$. As size of particles increases, their density decreases [24].

In this investigation, we have utilized Newton's method of differential corrections [25] [26] to compute numerically the halo orbits at the Lagrangian points L1 and L2 of the Sun-Mars system for different values of solar radiation pressure. Comparison of the size, shape, location and time period of L1 and L2 halo orbits of Sun-Mars system with respect to radiation pressure is made and some interesting results are drawn. This study will be useful for interplanetary low-energy transfer missions from Earth to Mars through Lagrangian points L1 and L2 using the stable and unstable manifolds.

\section{Equations of Motion}

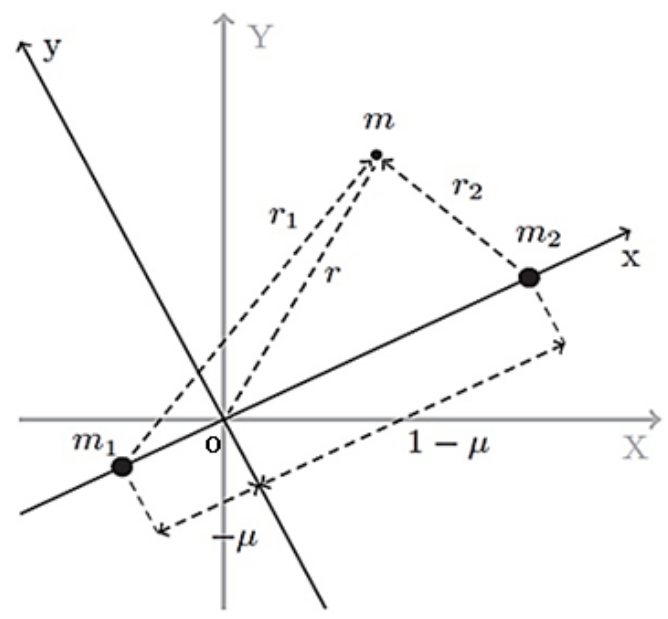

Figure 1. Diagram of the circular restricted three-body problem in normalized units $\left(\mu=\frac{m_{2}}{m_{1}+m_{2}}\right)$.

$r_{1}, r_{2}$ and $r$ are the positions of three bodies with masses $m_{1}$ (more massive source of radiation, Sun), $m_{2}$ (Planet) and $m$ (spacecraft or satellite). The origin $\mathrm{O}$ of the co-ordinate system is the barycentre of 
the two primaries with the more massive primary lying to the left of the origin and the smaller primary to the right as shown in Fig 1. For scaling purpose, the distance between the two primaries is taken as unity, the sum of masses of the primaries $\left(m_{1}+m_{2}\right)$ is assumed unity, and the gravitational constant is unity. Then the orbital mean motion of the primaries becomes unity. The non-dimensional mass ratio $(\mu)$ is defined as the ratio of the mass of the smaller primary to the sum of masses of the primaries i.e.

$$
\mu=m_{2} /\left(m_{1}+m_{2}\right) \text {. }
$$

The parameter $\mu$ defines the position of the larger and smaller primaries as $(-\mu)$ and $(1-\mu)$, respectively. Utilizing [1] [15] [16], the three-dimensional equations of motion are:

$$
\begin{gathered}
\ddot{x}-2 n \dot{y}=\frac{\partial U}{\partial x} \\
\ddot{y}+2 n \dot{x}=\frac{\partial U}{\partial y} \\
\ddot{z}=\frac{\partial U}{\partial z}
\end{gathered}
$$

where $U$ is the pseudo potential of the system and is given as

$$
U=\frac{n^{2}}{2}\left(x^{2}+y^{2}\right)+\frac{q(1-\mu)}{r_{1}}+\frac{\mu}{r_{2}}+\frac{\mu A 2}{2 r_{2}^{3}}
$$

In the above expression $r_{1}$ and $r_{2}$ are the position vectors of the particle from the more massive and smaller primary, respectively, i.e.,

$$
\begin{gathered}
r_{1 ?}=\sqrt{(x+\mu)^{2}+y^{2}+z^{2}} \\
r_{2 ?}=\sqrt{(x-(1-\mu))^{2}+y^{2}+z^{2}} \\
n^{2}=1+\frac{3}{2} A_{2}, \text { where } A 2=\frac{\left(A E^{2}-A P^{2}\right)}{5 R^{2}} .
\end{gathered}
$$

$A E$ and $A P$ are dimensional equatorial and polar radii of the smaller primary and $R$ is the distance between the primaries.

$q=1$ and $A 2=0$ correspond to the classical case of the restricted three-body problem.

\section{Liberation Points and Halo Orbits}

From the equations of motion Equations (1-3), it is apparent that an equilibrium solution exists relative to the rotating frame when the partial derivative of the pseudo potential function are all zero, i.e. $\Delta U=0$ or $\dot{x}, \dot{y}, \dot{z}=0$. These points correspond to the positions in the rotating frame at which the gravitational force and the centrifugal forces associated with the rotation of the synodic reference frame balance, with the result that a particle positioned at one of these points appears stationary in the synodic frame. Also for the collinear points, $y=z=0$.

Richardson [4] third-order approximation provides much qualitative insight. The approximate solution is sufficient for generating accurate motion near L1 and L2. Analytical approximation need to be combined with numerical techniques to generate a halo orbit accurate enough for mission design. In the present study, we use as the first guess for the differential correction process for obtaining halo orbits, the modified third-order approximation of Thurman and Worfolk [27] extended by Tiwary and Kushvah [23] for PRTBP using Lindstedt-Poincaré method, where more massive primary is the source of radiation and smaller primary is an oblate spheroid with its equatorial plane coincident with the plane of motion.

The method of differential correction is a powerful application of Newton's method that employs the state transition matrix (STM) to solve various boundary value problems. Differential correction method is used to determine the initial conditions of the halo orbits from the initial guess [25][28]. Taking 
advantage of the fact that halo orbits are symmetric about $x z$-plane, the initial state vector takes the form

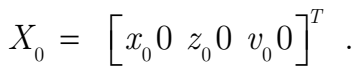

The equations of motion and State Transition Matrix are integrated numerically until the trajectory crosses the $x z$-plane again. The desired final condition is of the form

$$
X_{f}=\left[\begin{array}{lllll}
x_{f} & 0 & z_{f} & 0 & v_{f}
\end{array}\right]^{T}
$$

It is obtained by modifying the known parameters of the initial state vector.

The initial conditions obtained for the Sun-Mars system in the classical case for differential correction are:

For L1 halo orbit, when there is no solar radiation pressure $(q=1)$ and oblateness effect $(A 2=0)$,

$$
X=\left[\begin{array}{c}
0.994721415553 \\
0 \\
-0.000533438659 \\
0 \\
0.002051193403 \\
0
\end{array}\right]
$$

with a time period of 3.06765099769 i.e., 335.585 days in the classical restricted three-body problem (Fig. 2).

For L2 halo orbit, with $q=1$ and $A 2=0$,

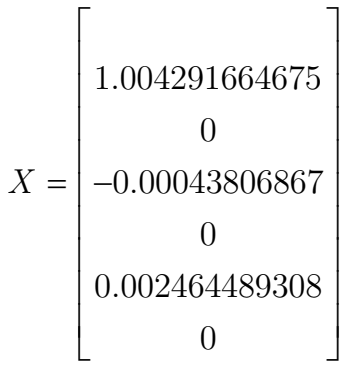

with a time period of 3.087915159265 i.e., 337.802 days in the classical restricted three-body problem (Fig. 2).

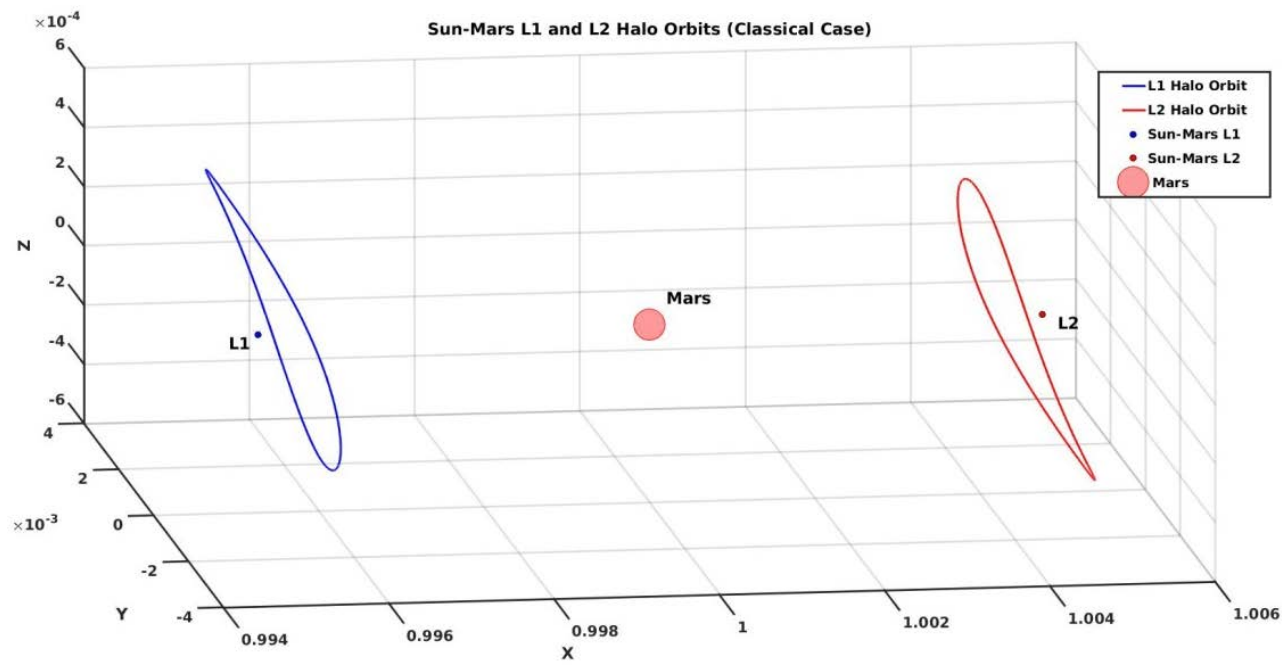

Figure 2. Sun-Mars L1 and L2 halo orbits. 


\subsection{Halo Orbits with Addition of Solar Radiation Pressure and Oblateness}

The halo orbits of Sun-Mars L1 and L2, obtained with analytical approximation and numerical solution using differential correction are shown in Figures 3 and 4 for $q=1,0.99$ and 0.98 . The oblateness term for Sun-Mars system $A 2=0.0000000001 \mathrm{e}-08$, taken as constant, is from [28]. Figures 3a to 3c provide the Sun-Mars L1 halo orbits with analytical and numerical solutions for different values of solar radiation pressure $q$ (1 to 0.98$)$. It is noted that the size of the orbit increases with radiation pressure. Figures 4a to 4c provide the Sun-Mars L2 halo orbits with analytical and numerical solutions for $q=1$ to 0.98 keeping constant value of $A 2$. It is noted that the size of the orbit decreases with radiation pressure.

\section{a) Sun-Mars L1 Halo Orbit when $\mathbf{q = 1 . 0}$}
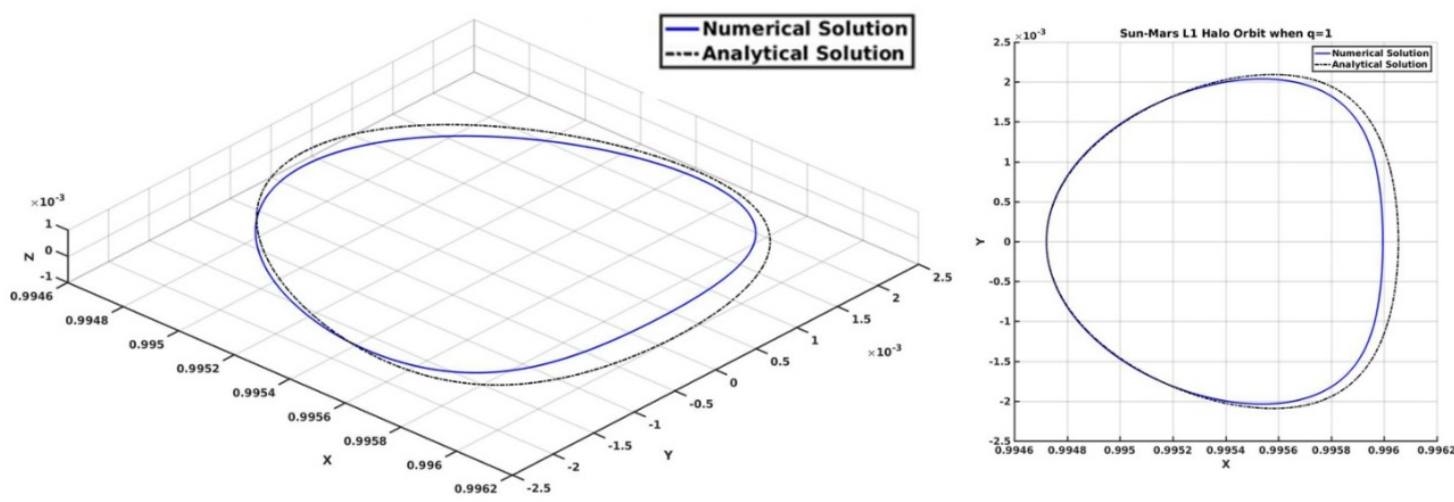

b) Sun-Mars L1 Halo Orbit when q=0.99
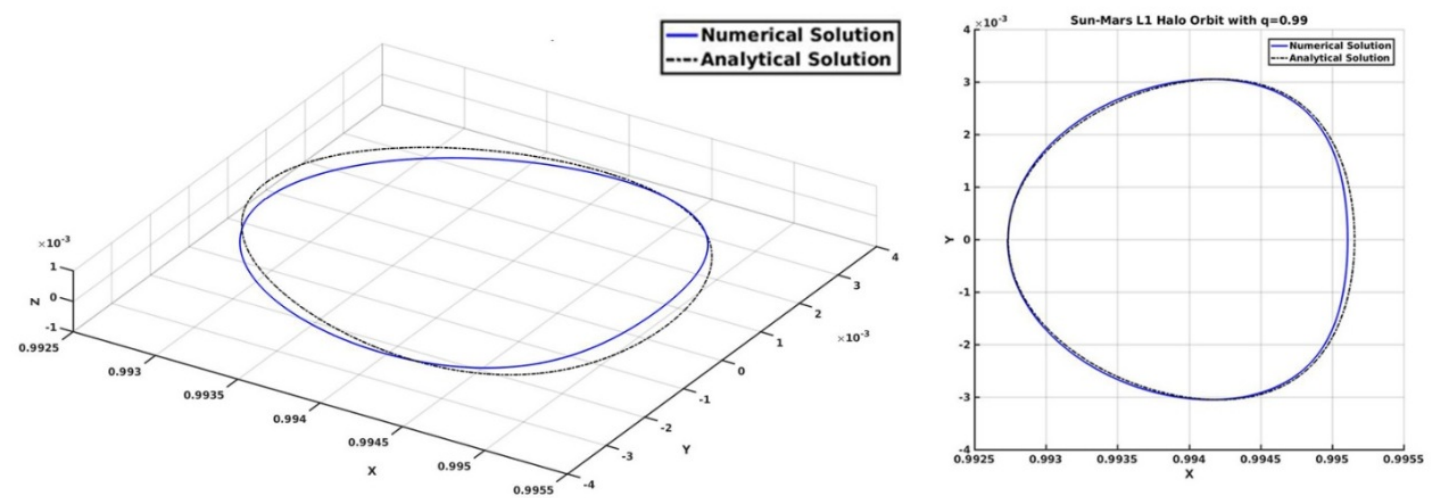

c) Sun-Mars L1 Halo Orbit when $\mathbf{q}=\mathbf{0 . 9 8}$
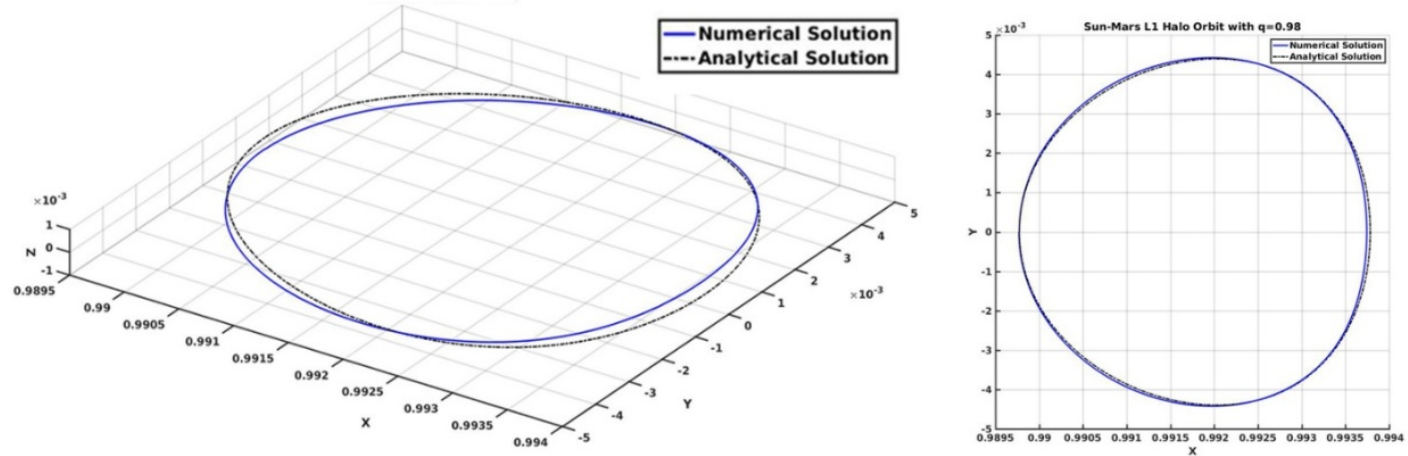

Figure 3. Sun-Mars L1 halo orbit with constant oblateness of Sun-Mars system

Though the oblateness $A 2$ for Sun-Mars system is constant, however to study the sensitivity of this 
term, we made a detailed study with different values of $A 2$ to generate the initial conditions. Similarly we have varied the value of $q$. Tables 1 and 2 in appendix I provide the initial conditions obtained by differential correction method with different values of solar radiation pressure and oblateness. These initial conditions have been utilized throughout the paper for generating the halo orbits at L1 and L2 by numerically integrating the equations of motion with Adams-Bashforth-Moulton multistep method with relative tolerance of $2.5 \mathrm{e}-10$ and absolute tolerance of $1 \mathrm{e}-14$.

\section{a) Sun-Mars L2 Halo Orbit when q=1}
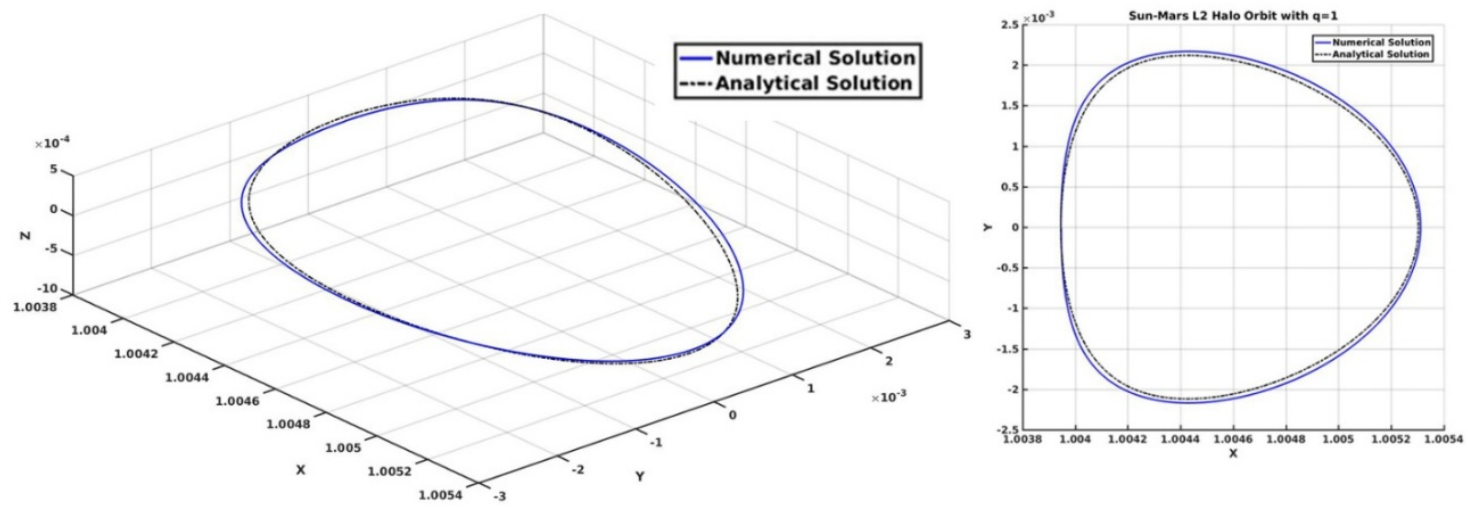

b) Sun-Mars L2 Halo Orbit when q=0.99
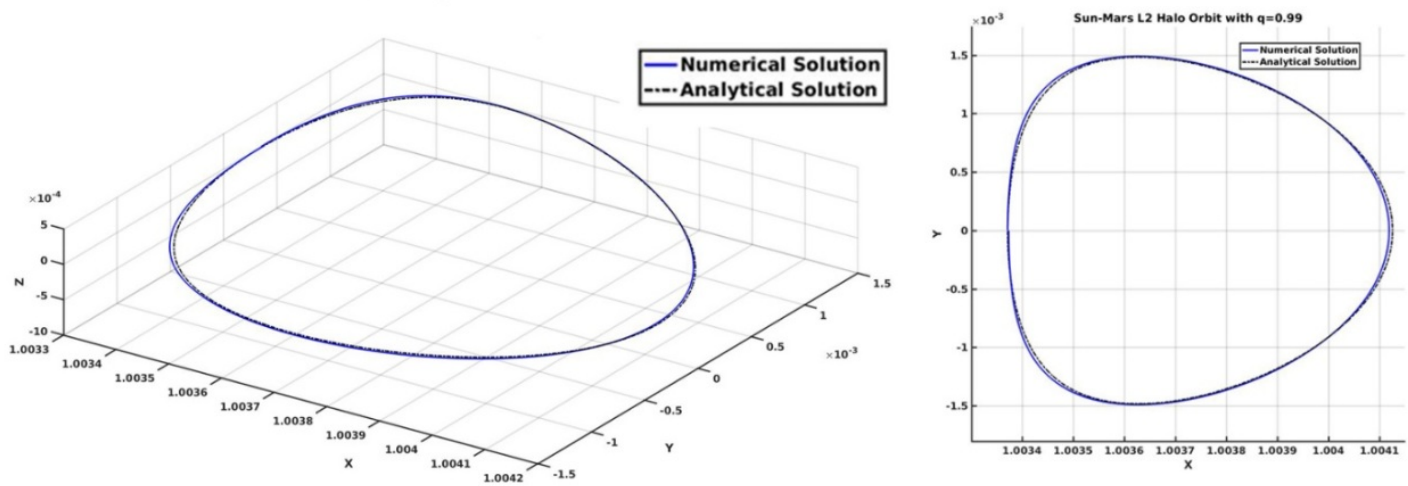

c)Sun-Mars L2 Halo Orbit when q=0.98
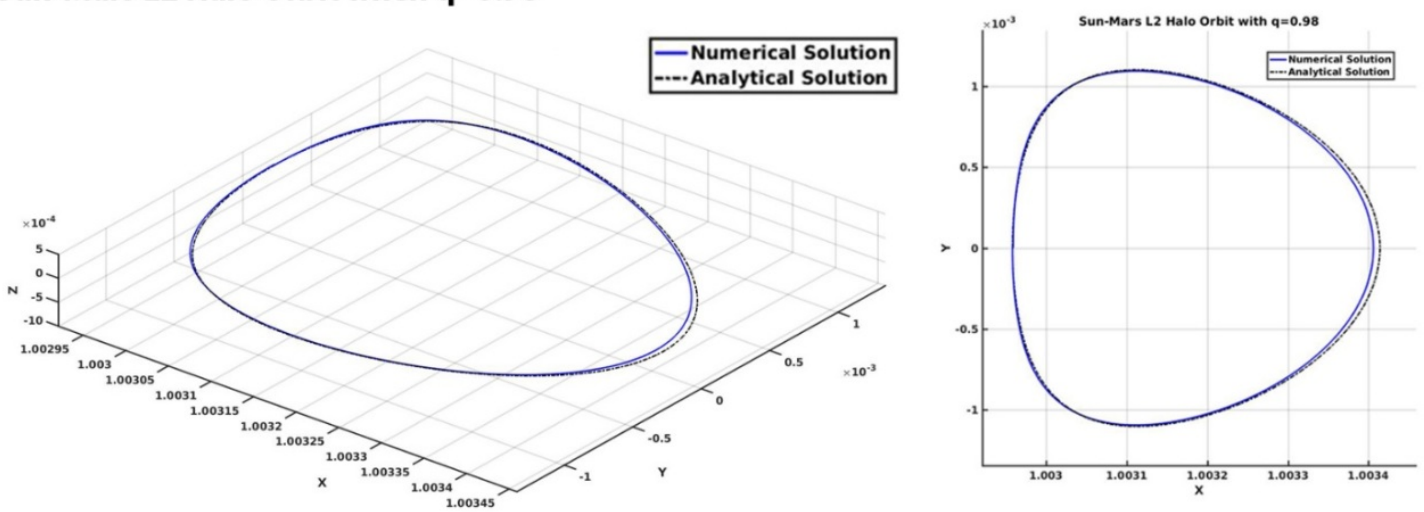

Figure 4. Sun-Mars L2 halo orbit with constant oblateness of Sun-Mars system.

\section{Variation in Size, Shape and Location of Sun-Mars L1 Halo Orbit}

The halo orbits around the Lagrangian point L1 are generated and plotted in Figures 6a to 6e with different values of $q$ and $A 2$ by using the initial conditions that are computed using differential 
correction method as in Table 1 of appendix I,. In Figures 5, 7 and 8, we have considered two values of $A 2$ as 0.000005 and 0.000010 which are significantly large to evaluate the effect of oblateness. The solar radiation pressure $q$ is varied from 1 to 0.97 . We study the effects of these perturbations on the shape, size and location of the halo orbits about L1 and L2. From Figures 5 and 8, we notice that in the absence of solar radiation pressure, oblateness significantly changes in the shape, size and location of the halo orbits. The dotted orbits are the halo orbits with $A 2=0.000010$. It may be noted that the size of halo orbits increases with the increase in solar radiation pressure and oblateness. However, the difference in the size of the halo orbit at L1 with oblateness reduces with increase in solar radiation pressure. It is also to be noted that the location of L1 halo orbit tends to move away from the smaller primary as the solar radiation pressure and oblateness increase. It can be seen from Figures 5, the L1 halo orbit elongates with increase in solar radiation pressure $q(q=1$ to 0.96$)$.

We have also considered the variation of $A 2$ from 0.000005 to 0.00003 and have varied the solar radiation pressure $q$ (1 to 0.96$)$ to study their effect on the shape, size, location and time period of halo orbits about L1 and L2. Figure 6 shows the halo orbits for different values of $q$ and $A 2$. Figure $6 \mathrm{f}$ shows the closer view of L1 halo orbit for $q=0.96$ with variation in $A 2$ from 0 to 0.00003 . It may be noted that these results on halo orbits with respect to radiation pressure are similar to the results of Bell [29], Baoyin and McInnes [30] and Eapen and Sharma [26].

\section{Variation in Size, Shape and Location of Sun-Mars L2 Halo Orbits}

The halo orbits about the Sun-Mars L2 are constructed in a similar way using the initial conditions in Table 2 of appendix I and are plotted in Figures 9a to 9e. In the absence of solar radiation pressure, the oblateness effect is almost similar as on L1 halo orbits. From Figures 6a to 6e and Figures 9a to 9e, on the contrary to the Sun-Mars L1 halo orbits, it can be noted that as the solar radiation pressure increases $(q=1.0$ to 0.96 ), the L2 halo orbits move towards the smaller primary and shrink about the Lagrangian point L2. As may be noted from Figure 7, with the increase in solar radiation pressure $(q=1$ to 0.96), the oblateness effect on the L2 halo orbit is significant. The oblateness effect increases the size of the halo orbit for corresponding value of $q$ and the orbit moves away from the smaller primary.

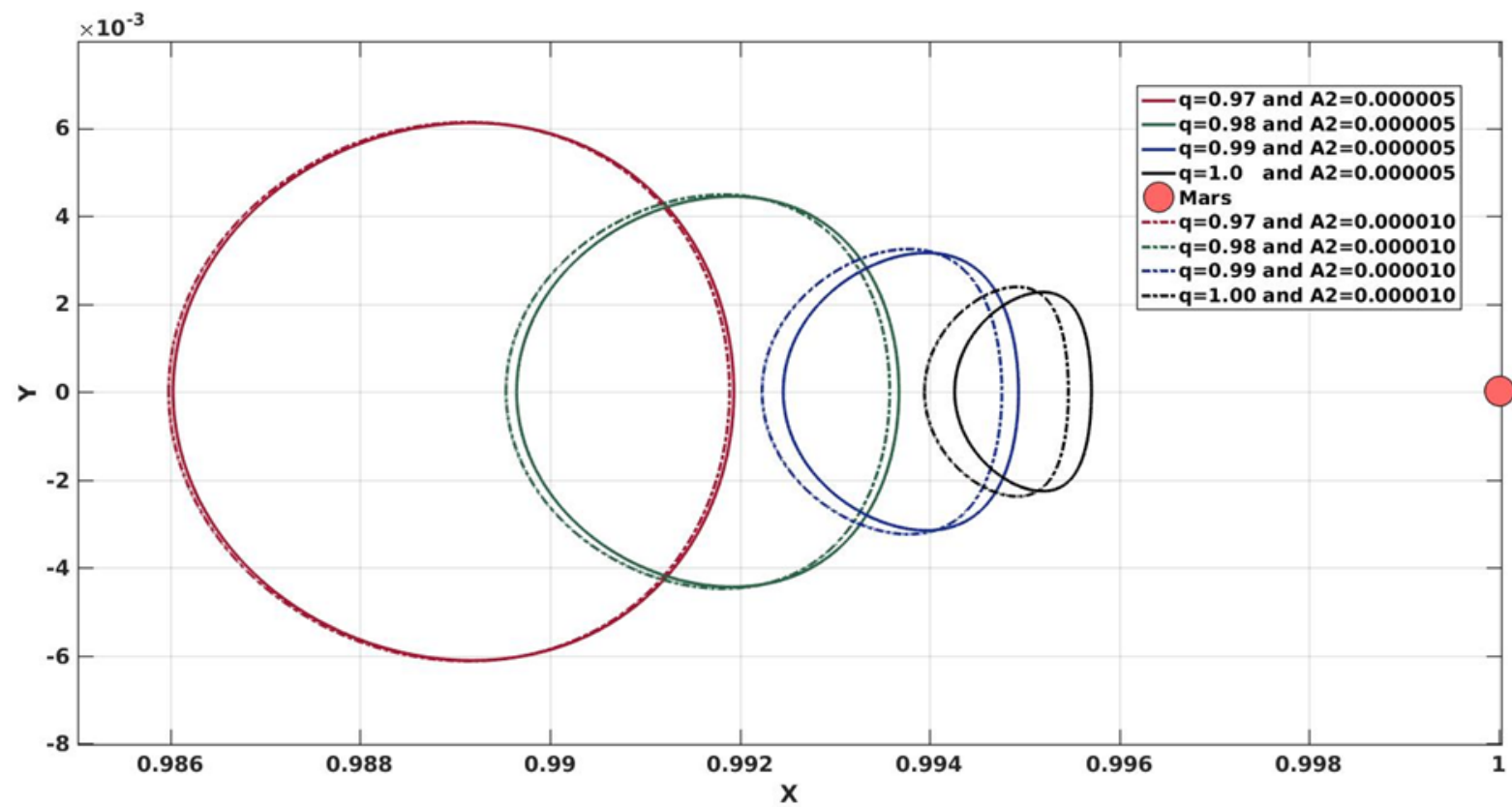

Figure 5. Variation of Sun-Mars L1 halo orbits with $q$ and $A 2$

Figure 8 shows the L1 and L2 halo orbits with different values of solar radiation pressure and oblateness. For a particular value of radiation pressure, the oblateness effect on the halo orbits L1 and 
L2 makes the orbit move away from the smaller primary.

Figures 9a to 9e show the effect of oblateness on halo orbits with constant $q$. It increases the size of the halo orbits and the orbits move away from the smaller primary. As radiation pressure increases, the size of the halo orbits decreases and moves towards the smaller primary.
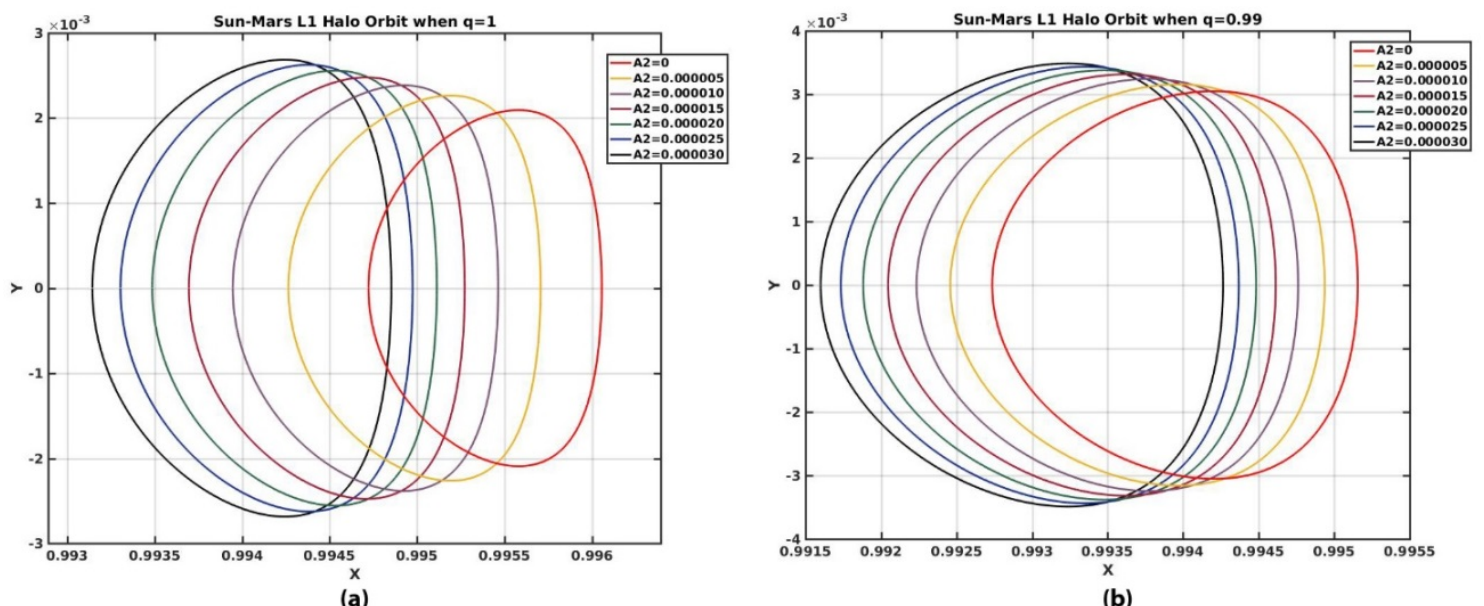

(b)

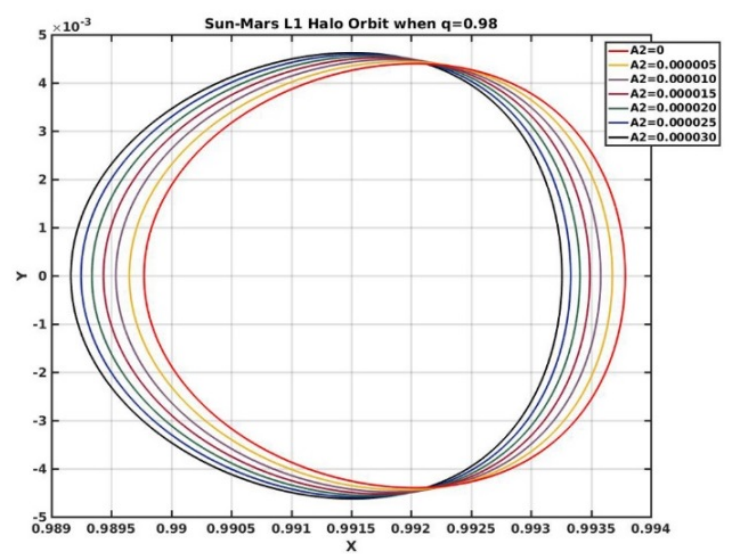

(c)

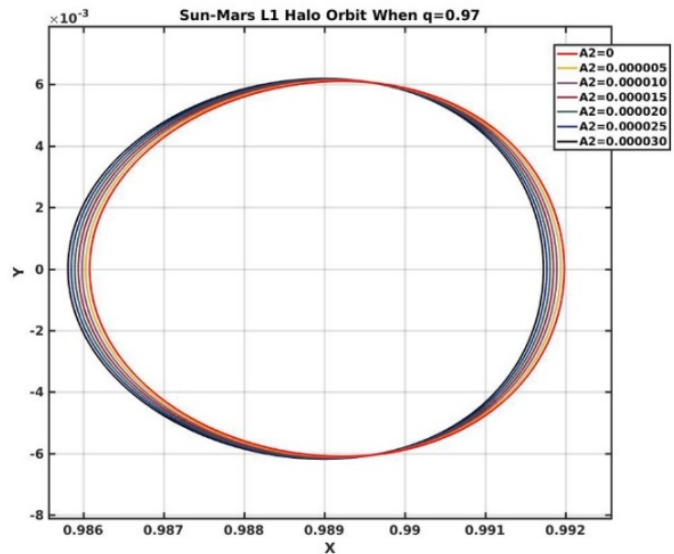

(d)

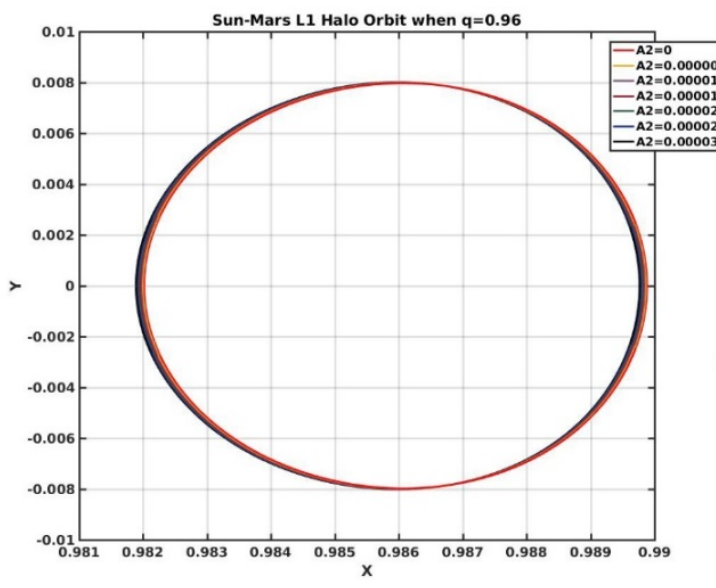

(e)

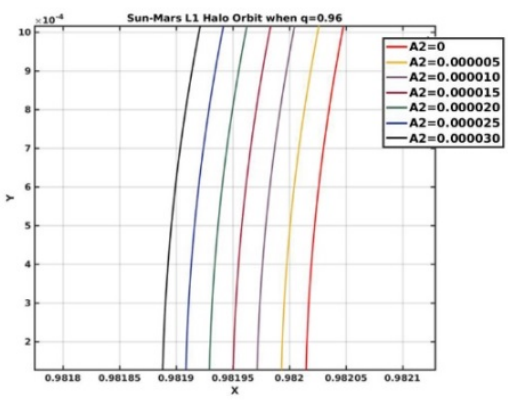

(f)

Figure 6. Sun-Mars L1 halo orbits for a) $q=1$ b) $q=0.99$ c) $q=0.98$ d) $q=0.97$ e) $q=0.96$ f) closer view of figure in $\mathrm{e}$. 


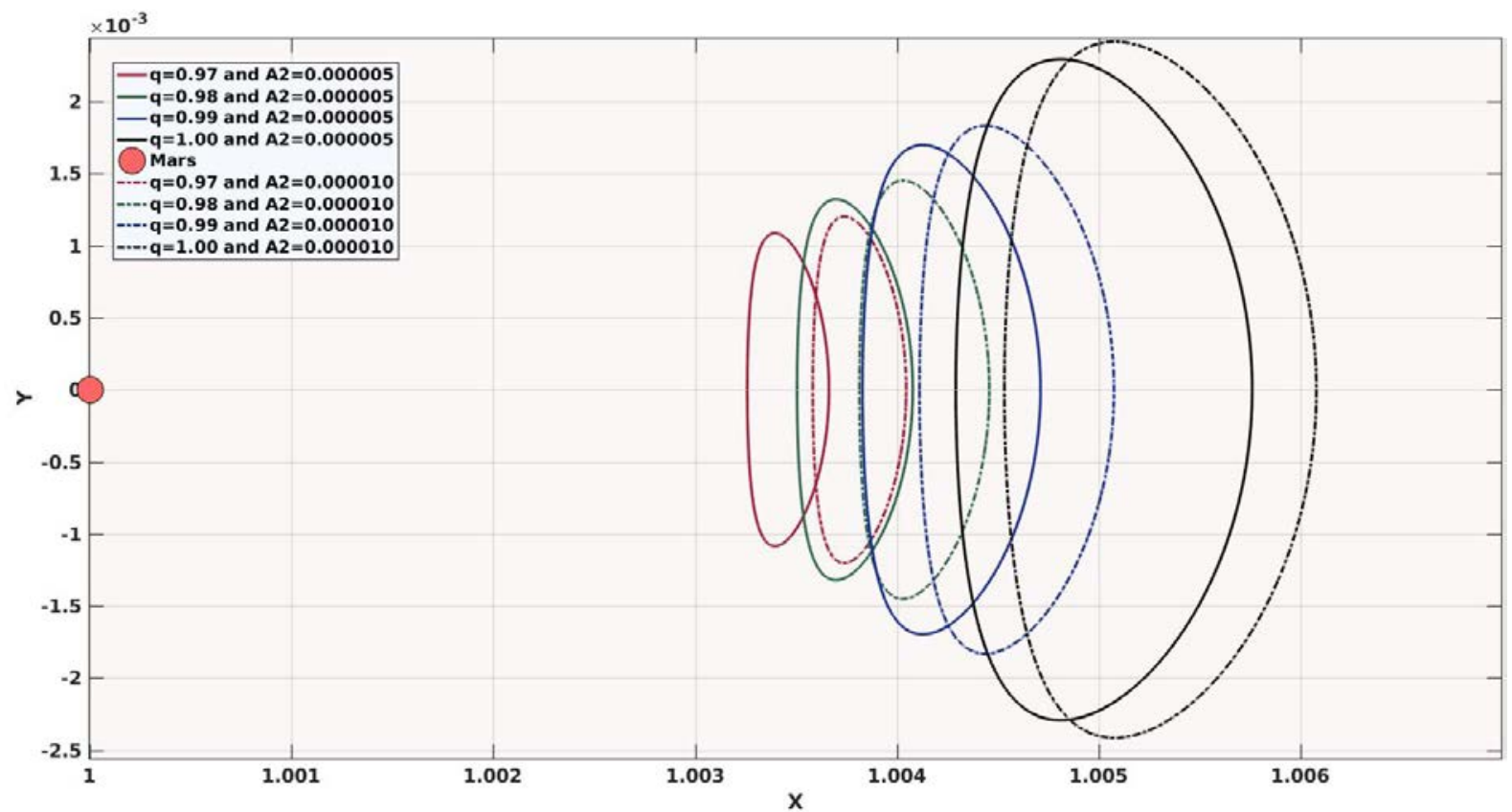

Figure 7. Variation of Sun-Mars L2 halo orbits with $q$ and $A 2$.

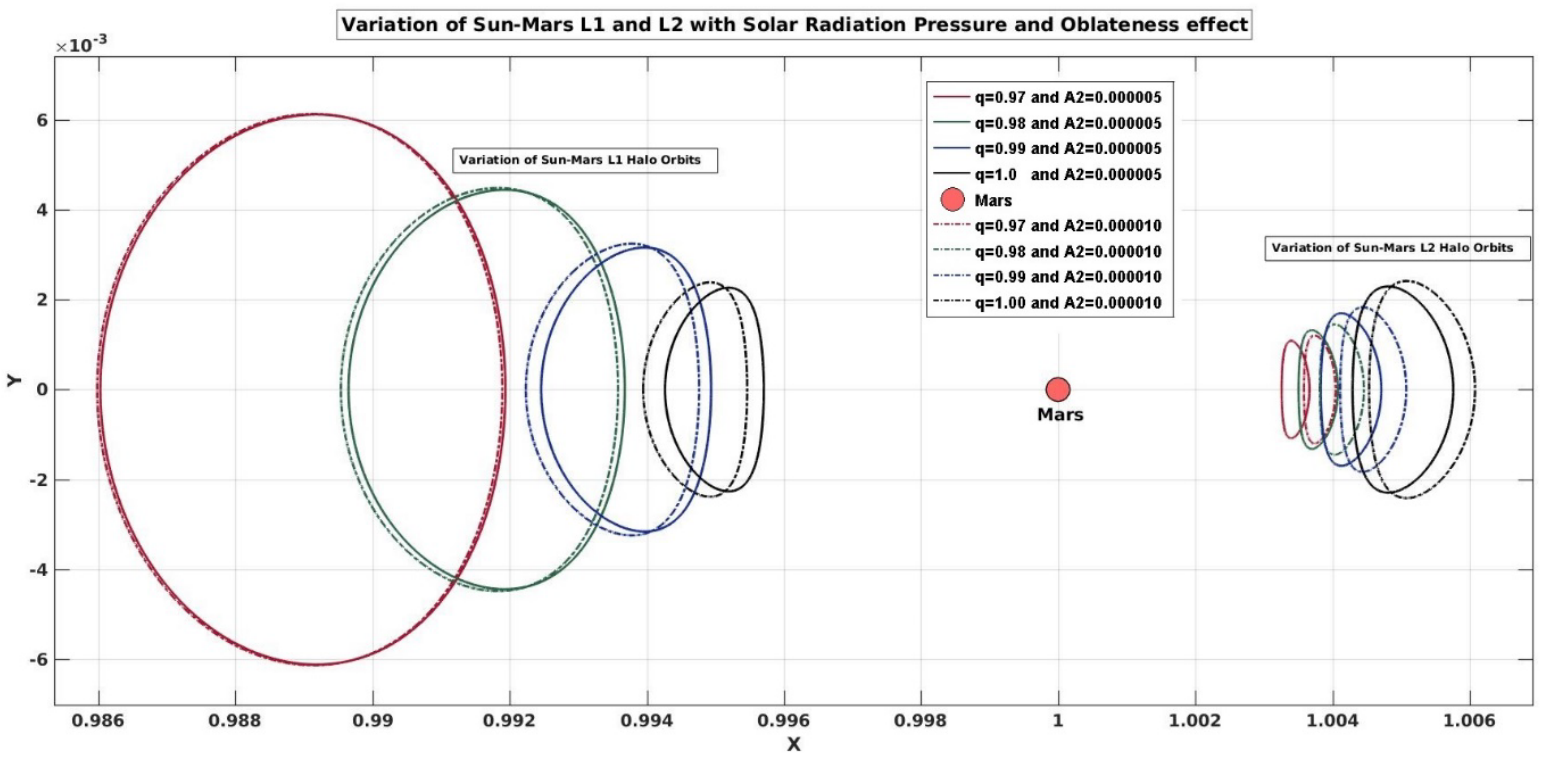

Figure 8. Variation of Sun-Mars both L1 and L2 halo orbits with $q$ and $A 2$. 


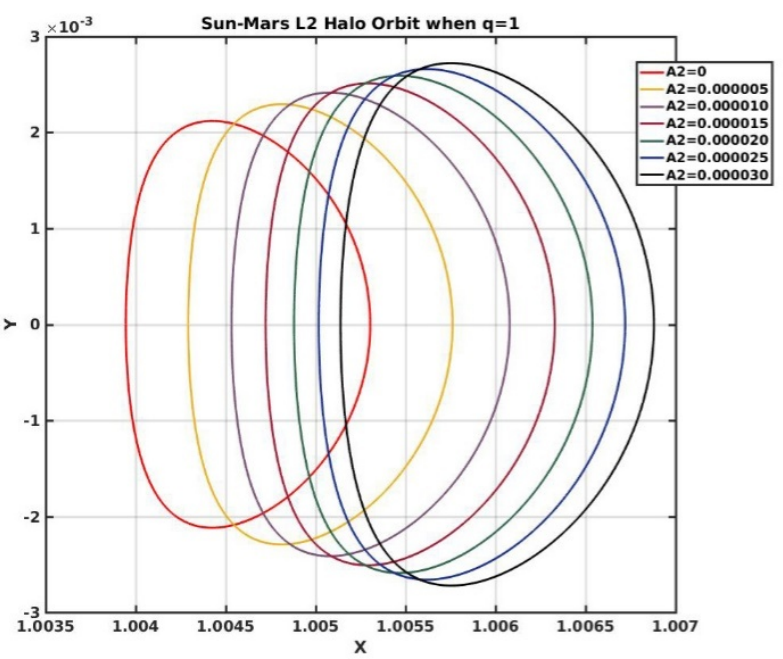

(a)

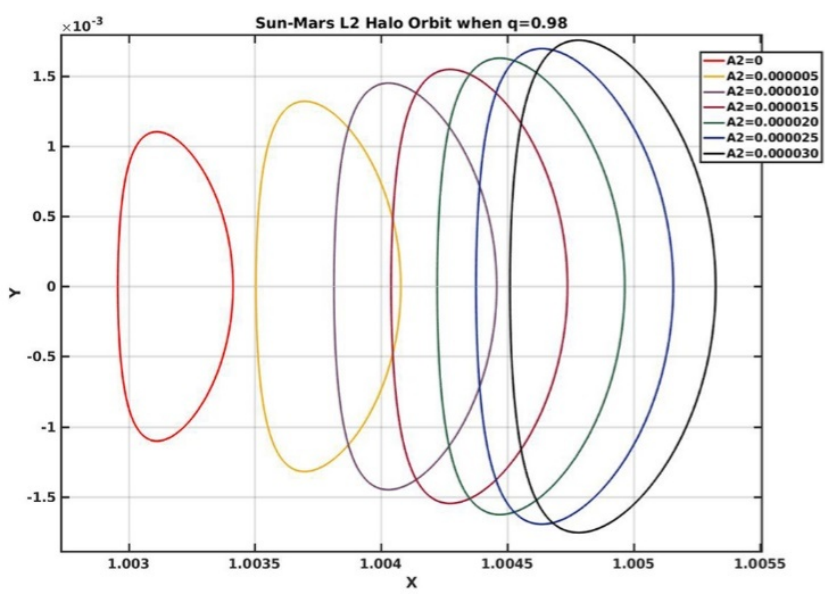

(c)

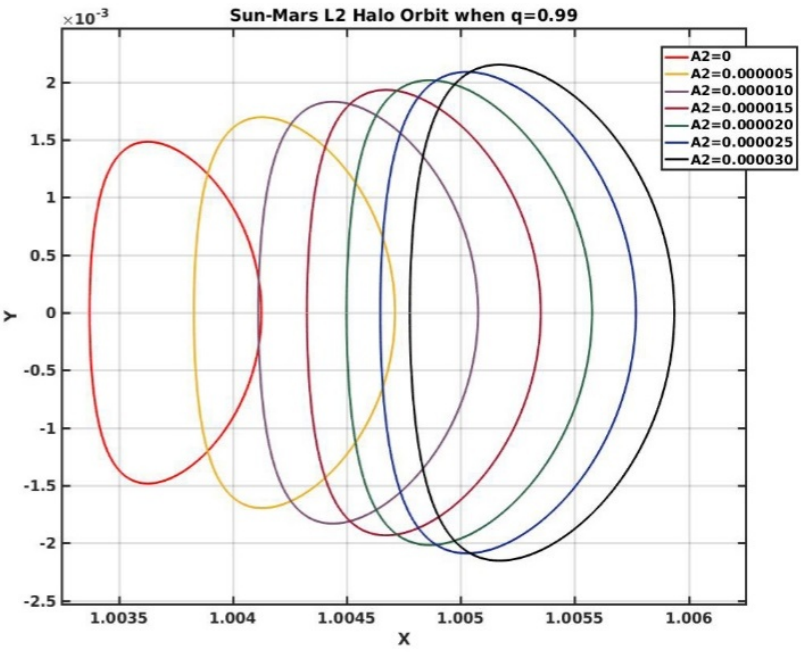

(b)

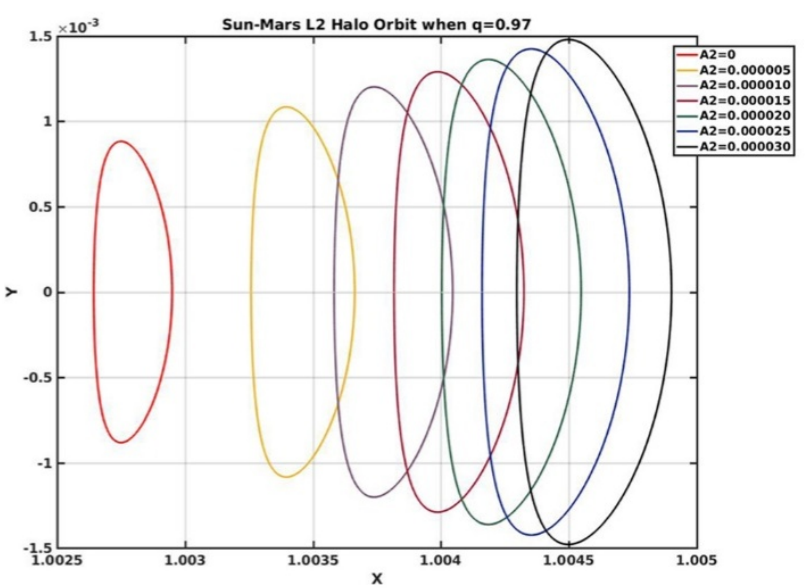

(d)

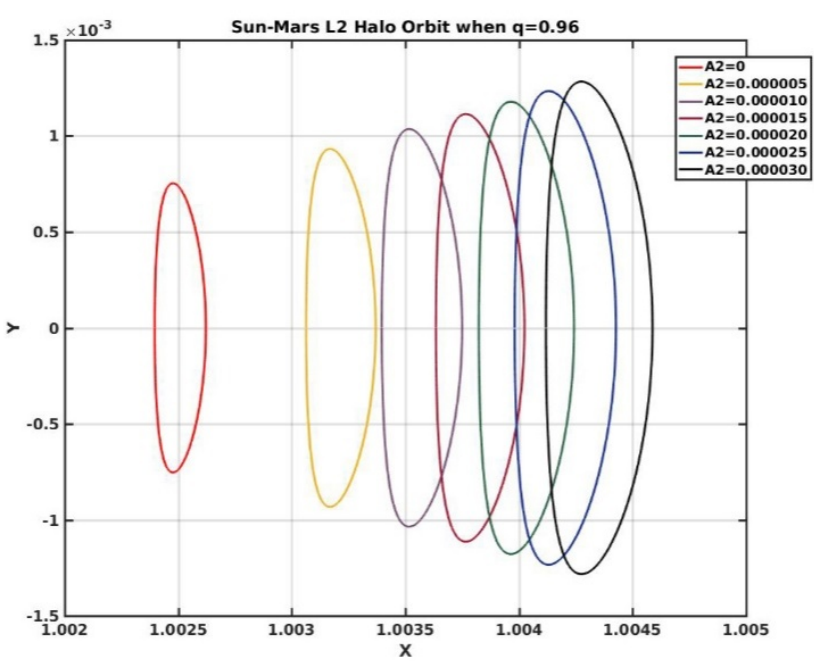

(e)

Figure 9. Sun-Mars L2 halo orbits for a) $q=1$ b) $q=0.99$ c) $q=0.98$ d) $q=0.97$ e) $q=0.96$. 


\section{Variation in Time Period with Solar Radiation Pressure and Oblateness}

Tables 1 and 2 in the appendix display the change in time period of L1 and L2 halo orbits with increase in $\varepsilon$ from 0 to 0.1 ( $q=1$ to $q=0.9$ ) for different values of $A 2$. It is depicted in Figures 10 and 11 . It is noticed that with increase in solar radiation pressure $(q=1$ to 0.96$)$, the time period of L1 halo orbit increases (Figure 10). We have also found that the increase in $A 2$ decreases the time period for corresponding values of $q$. Likewise, as the solar radiation pressure increases $(q=1$ to 0.96$)$, the time period of L2 halo orbit decreases (Figure 11). However, the increase in $A 2$ increases the time period of L2 halo orbit for corresponding values of $q$.

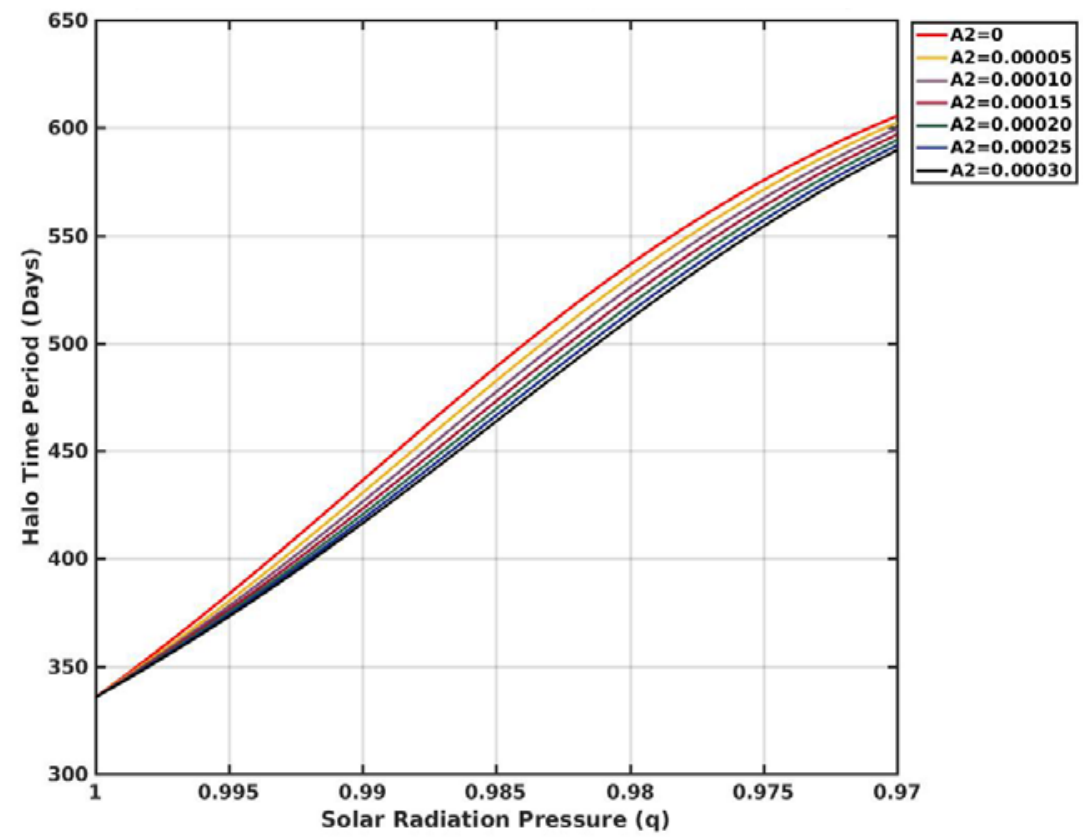

Figure 10. Variation of Sun-Mars L1 halo time period with $q$ and $A 2$.

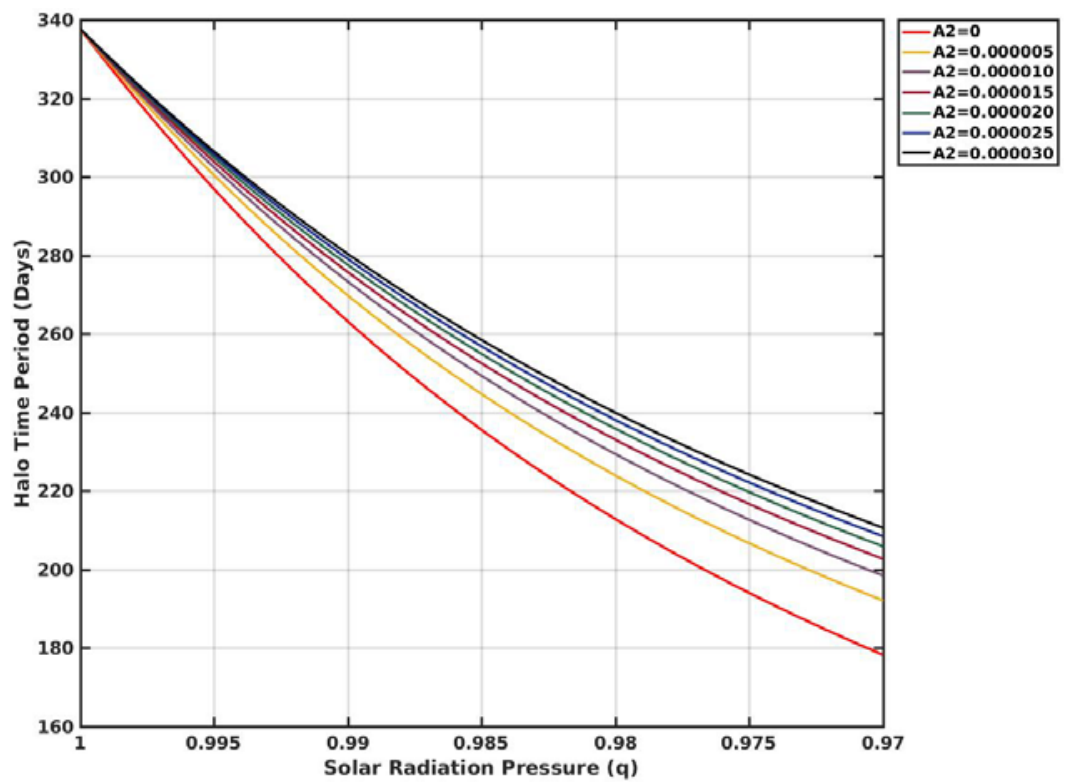

Figure 11. Variation of Sun-Mars L2 halo time period with $q$ and $A 2$. 


\section{Results and Conclusion}

The halo orbits about the Lagrangian points L1 and L2 of Sun-Mars system in the framework of photogravitational restricted three-body problem with oblateness are generated numerically by integrating the equations of motion. The initial conditions for numerical integration are found through analytical expressions of Tiwary and Kushvah [23] and differential correction method. Evolution of L1 and L2 halo orbits with respect to solar radiation pressure and oblateness are studied. It is found that the increase in solar radiation pressure at constant oblateness elongates the halo orbits at L1. They move towards the radiating body. At L2 the halo orbits shrink and move towards the smaller primary with increase in solar radiation pressure at constant oblateness. For constant radiation pressure, the increase in oblateness causes the location of L1 and L2 halo orbits to move away from the smaller primary. It is also noted that in the case of L1 halo orbit, the effect of oblateness is much less for higher values of radiation pressure. And in the case of L2 halo orbits, the effect of oblateness is higher for the higher values of radiation pressure. The time period of L1 halo orbits increases with increase in radiation pressure for constant oblateness and decreases with increase in oblateness for constant radiation pressure. However, this trend reverses for L2 halo orbits, i.e., the time period of L2 halo orbits decreases with increase in radiation pressure for constant oblateness and increases with increase in oblateness for constant radiation pressure.

Acknowledgements. The authors gratefully acknowledge ISRO Satellite Centre, Indian Space Research Organisation, Bangalore for the financial support through RESPOND project.

\section{References}

1. V. Szebehely, "Theory of Orbits. The Restricted Problem of Three Bodies." Academic press, New York, 1967.

2. R.W. Farquhar and A.A. Kamel, "Quasi-Periodic Orbits about the translunar libration point." Celestial Mechanics, vol. 7, pp. 458-473, 1973.

3. R.W. Farquhar, D.P Muhonen and D.L Richardson, "Mission design for a halo orbiter of the Earth." Journal of Spacecraft and Rockets, vol. 14, pp. 170-177, 1977.

4. D.L Richardson, "Analytic construction of periodic orbits about the collinear points." Celestial Mechanics, vol. 22, pp. 241-253, 1980.

5. M. Huber, R. Bonnet, D. Dale, M. Arduini, C. Fröhlich, V. Domingo and G. Whitcomb, "The History of the SOHO Mission", ESA Bulletin, vol. 86, pp. 25-35, 1996.

6. G. Go mez, A. Jorba, C. Sim o and J. Masdemont, "Dynamics and Mission Design near Libration Points Vol.III Advanced Method for Collinear Points." New Jersey: World Scientific, 2001.

7. G. Go mez, J. Llibre, R. Mart $i$ nez and C. Sim o, "Dynamics and Mission Design near Libration Points Vol.I Fundamentals: The Case of Collinear Libration Points." New Jersey: World Scientific, 2001.

8. R.R. Rausch, "Earth to Halo Orbits Transfer Trajectory", MS thesis, Purdue University, 2005.

9. M. Nakamiya, D.J. Scheeres, H. Yamakawa and M. Yoshikawa, "Analysis of capture trajectories into periodic orbits about liberation points", Journal of Guidance, Control and Dynamics, vol. 31, no. 5, pp. 1344-1351, 2008.

10. W.S. Koon, M. Lo, J.E. Marsden and Ross, S.D, "Dynamical Systems: The Three Body Problem and Space Mission Design." Interdisciplinary Applied Mathematics, Springer, New York, vol. 332, 315 pages, 2011.

11. J. Lu, M. Zang and Q. Lu, "Transfer Trajectory Design for Mars Exploration", International Journal of Astronomy and Astrophysics, vol. 3, pp. 5-16, 2013.

12. P. Nath and R.V. Ramanan, "Precise halo orbit design and optimal transfer to halo orbits from earth using differential evolution", Advances in Space Research, vol. 57, pp. 202-217, 2015.

13. V.V. Radzievskii, “The restricted problem of three bodies taking account of light pressure." Astron. Zh. vol. 27, no. 5, pp. 250, 1950.

14. Y.A. Chernikov, "The photogravitational restricted problem of three bodies." Astron. Zh. vol. 47, pp. 217-223, 1970 .

15. K.B. Bhatnagar and J.M. Chawla, "A study of the Lagrangian points in the photogravitational restricted three-body problem" Indian J. Pure Appl. Math. vol. 10, no. 11, pp. 1443-1451, 1979.

16. R.K. Sharma, "The linear stability of libration points of the photogravitational restricted three-body problem when the smaller primary is an oblate spheroid." Astrophys. Space Sci. vol. 135, no. 2, pp. 271-281, 1987.

17. A.L. Kunitsyn and A.T. Tureshbaev, "On the collinear libration points in the photogravitational three-body 
problem." Celest. Mech. vol. 35 no. 2, pp. 105-112, 1985.

18. L.G. Lukyanov, "On the family of the libration points in the restricted photogravitational three-body problem." Astron. Zh. vol. 65, pp. 422-432, 1988.

19. J.V. Breakwell and J.V. Brown, "The Halo Family of 3-Dimensional Periodic Orbits in the Earth-Moon Restricted Three Body Problem", Celestial Mechanics, vol. 20, pp. 389-404, 1979.

20. K.C. Howell, "Three-Dimensional Periodic 'Halo' Orbits in the Restricted Three-Body Problem", Ph.D. Dissertation, Department of Aeronautics and Astronautics, Stanford University, Stanford, California, 1983.

21. K.C. Howell, "Three-Dimensional, Periodic 'Halo' Orbits", Celestial Mechanics, vol. 32, pp.53-71, 1984.

22. K.C. Howell, B.T. Barden and M.W. Lo, "Application of Dynamical Systems Theory to Trajectory Design for a Libration Point Mission", Journal of the Astronautical Sciences, vol. 45, pp. 161-178, 1997.

23. R.D. Tiwary and B.S. Kushvah, "Computation of halo orbits in the photogravitational Sun-Earth system with oblateness." Astrophys. Space Sci., vol. 357, no. 73, pp. 1-16, 2015.

24. E. Grün, B.A.S. Gustafson, S. Dermott and H. Fechtig, "Interplanetary Dust." Springer, Berlin, 2001.

25. J.D. Mireles, "The state transition matrix and method of differential corrections." Celestial Mechanics: Problem Set 2, Rutgers University, 51 pages, 2006.

26. R.T. Eapen and R.K. Sharma, "A study of halo orbits at the Sun-Mars L1 Lagrangian point in the photogravitational restricted three-body problem." Astrophys. Space Sci., vol. 352, pp. 437-441, 2014.

27. R. Thurman and P.A. Worfolk, "The geometry of halo orbits in the circular restricted three-body problem." Technical report, University of Minnesota, Minneapolis, Tech. Rep. GCG 95.1996.

28. A. McInnes, "An Introduction to Liberation Point Orbits", Notes, 19 pages, 2009. coweb.elec.canterbury.ac.nz/cda/uploads/mcinnes-intro_to_libration_point_orbits.pdf.

29. R.K. Sharma and P.V. Subba Rao, "Collinear equilibria and their characteristic exponents in the restricted three-body problem when the primaries are oblate spheroids", Celestial Mechanics, vol. 12, pp. 189-201, 1975.

30. J.L. Bell, "The impact of solar radiation pressure on Sun-Earth L1 libration point orbits." M.S. thesis, Purdue Univ., USA, 1991.

31. H. Baoyin and C.R. McInnes, "Solar sail halo orbits at the Sun-Earth artificial L1 point", Celestial Mechanics and Dynamical Astronomy, vol. 94, pp. 155-171, 2006.

\section{Appendix I}

Table 1a. Sun-Mars L1 halo orbit initial conditions and time period at $A 2=0$.

\begin{tabular}{ccccccc}
\hline $\mathbf{q}$ & $\mathbf{T}$ & $\mathbf{T} / \mathbf{2}$ & $\mathbf{x}$ & $\mathbf{z}$ & $\dot{\mathbf{y}}$ & Tp in Days \\
\hline $\mathbf{1}$ & 3.0676510 & 1.5338254988450 & 0.994721415553 & 0.000533438659 & 0.002051193403 & 335.585387804623 \\
$\mathbf{0 . 9 9}$ & 3.9882184 & 1.9941092143970 & 0.992735131944 & 0.000536130642 & 0.003020420092 & 436.290773978052 \\
$\mathbf{0 . 9 8}$ & 4.9044121 & 2.4522060769610 & 0.989771489489 & 0.000523136009 & 0.004398865484 & 536.517699003778 \\
$\mathbf{0 . 9 7}$ & 5.5342487 & 2.7671243482410 & 0.986077784635 & 0.000506577987 & 0.006129200561 & 605.418607401773 \\
$\mathbf{0 . 9 6}$ & 5.8776863 & 2.9388431513720 & 0.982014339537 & 0.000495259393 & 0.008008257781 & 642.988931526185 \\
$\mathbf{0 . 9 5}$ & 6.0522484 & 3.0261242189420 & 0.977779737744 & 0.000488730861 & 0.009922204509 & 662.085139621914 \\
$\mathbf{0 . 9 4}$ & 6.1430516 & 3.0715258295030 & 0.973449467103 & 0.000484942263 & 0.011849931937 & 672.018549321307 \\
$\mathbf{0 . 9 3}$ & 6.19302290 & 3.0965114485430 & 0.969053543181 & 0.000482582190 & 0.013791581880 & 677.485148136637 \\
$\mathbf{0 . 9 2}$ & 6.22223222 & 3.1111161077780 & 0.964606362890 & 0.000480974662 & 0.015747494376 & 680.680498740073 \\
$\mathbf{0 . 9 1}$ & 6.24026129 & 3.1201306429840 & 0.960115723333 & 0.000479779591 & 0.017716834996 & 682.652787175271 \\
$\mathbf{0 . 9}$ & 6.25192161 & 3.1259608050790 & 0.955586064487 & 0.000478820728 & 0.019698467611 & 683.928367225992 \\
\hline
\end{tabular}


Table 1b. Sun-Mars L1 halo orbit initial conditions and time period at $A 2=0.000005$.

\begin{tabular}{ccccccc}
\hline $\mathbf{q}$ & $\mathbf{T p}$ & $\mathbf{T p} / \mathbf{2}$ & $\mathbf{x}$ & $\mathbf{z}$ & $\dot{\mathbf{y}}$ & $\mathbf{T p}$ in Days \\
\hline $\mathbf{1}$ & 3.06750885 & 1.533754422617 & 0.994264003213 & 0.000533165976 & 0.002220172713 & 335.569837050389 \\
$\mathbf{0 . 9 9}$ & 3.93523513 & 1.967617563120 & 0.992457990757 & 0.000536289329 & 0.003124142346 & 430.494670657037 \\
$\mathbf{0 . 9 8}$ & 4.85150017 & 2.425750087164 & 0.989649032237 & 0.000524208121 & 0.004441312481 & 530.729398051487 \\
$\mathbf{0 . 9 7}$ & 5.50649887 & 2.753249436421 & 0.986029733449 & 0.000507386520 & 0.006142832345 & 602.382918096042 \\
$\mathbf{0 . 9 6}$ & 5.86590733 & 2.932953665246 & 0.981992635570 & 0.000495651571 & 0.008014700148 & 641.700371982057 \\
$\mathbf{0 . 9 5}$ & 6.04727592 & 3.023637957809 & 0.977767742477 & 0.000488906848 & 0.009926659977 & 661.541171023816 \\
$\mathbf{0 . 9 4}$ & 6.14080423 & 3.070402115693 & 0.973441648735 & 0.000485024025 & 0.011853328489 & 671.772692191321 \\
$\mathbf{0 . 9 3}$ & 6.19191772 & 3.095958858984 & 0.969047754898 & 0.000482622619 & 0.013794284164 & 677.364247172614 \\
$\mathbf{0 . 9 2}$ & 6.22164244 & 3.110821220700 & 0.964601648822 & 0.000480995937 & 0.015749747011 & 680.615980452516 \\
$\mathbf{0 . 9 1}$ & 6.23992191 & 3.119960953472 & 0.960111617004 & 0.000479791406 & 0.017718799729 & 682.615660839365 \\
$\mathbf{0 . 9}$ & 6.2517122 & 3.125856098339 & 0.955582322739 & 0.000478827580 & 0.019700245819 & 683.905458458355 \\
\hline
\end{tabular}

Table 1c. Sun-Mars L1 halo orbit initial conditions and time period at $A 2=0.000010$.

\begin{tabular}{|c|c|c|c|c|c|c|}
\hline $\mathbf{q}$ & $\mathbf{T p}$ & $\mathbf{T p} / 2$ & $\mathbf{x}$ & $\mathbf{z}$ & $\dot{\mathbf{y}}$ & Tp in Days \\
\hline 1 & 3.06749655 & 1.533748273596 & 0.993946023502 & 0.000533006906 & 0.002337953676 & 335.568491707150 \\
\hline 0.99 & 3.89711087 & 1.948555435473 & 0.992234941516 & 0.000536366570 & 0.003207690735 & 426.324071391595 \\
\hline 0.98 & 4.8062213 & 2.403110649209 & 0.989537017980 & 0.000525089915 & 0.004480571643 & 525.776119747210 \\
\hline 0.97 & 5.48036092 & 2.740180462426 & 0.985983085787 & 0.000508139163 & 0.006156213656 & 599.523559772713 \\
\hline 0.96 & 5.85439195 & 2.927195973539 & 0.981971187110 & 0.000496033300 & 0.008021033538 & 640.440647713678 \\
\hline 0.95 & 6.04235029 & 3.021175146597 & 0.977755813125 & 0.000489080836 & 0.009931063528 & 661.002333029344 \\
\hline 0.94 & 6.13856713 & 3.069283564781 & 0.973433850673 & 0.000485105332 & 0.011856705150 & 671.527964651130 \\
\hline 0.93 & 6.19081541 & 3.095407705958 & 0.969041973787 & 0.000482662921 & 0.013796979006 & 677.243660507481 \\
\hline 0.92 & 6.22105365 & 3.110526826229 & 0.964596937648 & 0.000481017169 & 0.015751996690 & 680.551569942460 \\
\hline 0.91 & 6.23958293 & 3.119791464041 & 0.960107511998 & 0.000479803203 & 0.017720763189 & 682.578578279143 \\
\hline 0.9 & 6.25150297 & 3.125751484218 & 0.955578581660 & 0.000478834425 & 0.019702023439 & 683.882569954703 \\
\hline
\end{tabular}


Table 1d. Sun-Mars L1 halo orbit initial conditions and time period at $A 2=0.000015$.

\begin{tabular}{ccccccc}
\hline $\mathbf{q}$ & $\mathbf{T p}$ & $\mathbf{T p} / \mathbf{2}$ & $\mathbf{x}$ & $\mathbf{z}$ & $\dot{\mathbf{y}}$ & Tp in Days \\
\hline $\mathbf{1}$ & 3.06754723 & 1.533773616023 & 0.993695480364 & 0.000532895785 & 0.002430913218 & 335.574036371911 \\
$\mathbf{0 . 9 9}$ & 3.86752041 & 1.933760204060 & 0.992045894751 & 0.000536404937 & 0.003278544553 & 423.087025538078 \\
$\mathbf{0 . 9 8}$ & 4.76676256 & 2.383381282402 & 0.989433479781 & 0.000525831093 & 0.004517178323 & 521.459535353668 \\
$\mathbf{0 . 9 7}$ & 5.45567374 & 2.727836869835 & 0.985937735118 & 0.000508842021 & 0.006169363547 & 596.822907508595 \\
$\mathbf{0 . 9 6}$ & 5.84313006 & 2.921565031954 & 0.981949983988 & 0.000496405030 & 0.008027266789 & 639.2086555080272 \\
$\mathbf{0 . 9 5}$ & 6.03747085 & 3.018735424024 & 0.977743948252 & 0.000489252861 & 0.009935417132 & 660.468546593677 \\
$\mathbf{0 . 9 4}$ & 6.13634028 & 3.068170140419 & 0.973426072684 & 0.000485186187 & 0.011860062282 & 671.284358747833 \\
$\mathbf{0 . 9 3}$ & 6.18971597 & 3.094857984086 & 0.969036199805 & 0.000482703097 & 0.013799666475 & 677.123386964039 \\
$\mathbf{0 . 9 2}$ & 6.22046585 & 3.110232923238 & 0.964592229359 & 0.000481038358 & 0.015754243430 & 680.487266963219 \\
$\mathbf{0 . 9 1}$ & 6.23924435 & 3.119622174380 & 0.960103408311 & 0.000479814984 & 0.017722725378 & 682.541539426562 \\
$\mathbf{0 . 9}$ & 6.25129393 & 3.125646962608 & 0.955574841251 & 0.000478841262 & 0.019703800473 & 683.859701691623 \\
\hline
\end{tabular}

Table 2a. Sun-Mars L2 halo orbit initial conditions and time period at $A 2=0$.

\begin{tabular}{ccccccc}
\hline $\mathbf{q}$ & $\mathbf{T p}$ & $\mathbf{T p} / \mathbf{2}$ & $\mathbf{x}$ & $\mathbf{z}$ & $\dot{\mathbf{y}}$ & Tp in Days \\
\hline $\mathbf{1}$ & 3.087446 & 1.543723267619 & 1.003945241736 & 0.000437917046 & 0.002279076491 & 337.750918743283 \\
$\mathbf{0 . 9 9}$ & 2.4064763 & 1.203238154353 & 1.003371368838 & 0.000446735642 & 0.001572461880 & 263.256245872774 \\
$\mathbf{0 . 9 8}$ & 1.9464034 & 0.973201741441 & 1.002958021743 & 0.000454787246 & 0.001155002880 & 212.926623047760 \\
$\mathbf{0 . 9 7}$ & 1.6302149 & 0.815107468002 & 1.002642969872 & 0.000460252105 & 0.000917475874 & 178.337207171248 \\
$\mathbf{0 . 9 6}$ & 1.4033900 & 0.701695033458 & 1.002396050032 & 0.000463757932 & 0.000778992641 & 153.523722288422 \\
$\mathbf{0 . 9 5}$ & 1.2338309 & 0.616915453545 & 1.002198047827 & 0.000466040145 & 0.000694982401 & 134.974814199067 \\
$\mathbf{0 . 9 4}$ & 1.1025907 & 0.551295362311 & 1.002035866739 & 0.000467581500 & 0.000641962106 & 120.617806976961 \\
$\mathbf{0 . 9 3}$ & 0.9980572 & 0.499028608611 & 1.001900456205 & 0.000468667801 & 0.000607291093 & 109.182373922325 \\
$\mathbf{0 . 9 2}$ & 0.9128093 & 0.456404687868 & 1.001785474522 & 0.000469466210 & 0.000583915711 & 99.856694447444 \\
$\mathbf{0 . 9 1}$ & 0.8419166 & 0.420958319853 & 1.001686398043 & 0.000470076109 & 0.000567749459 & 92.101390362633 \\
$\mathbf{0 . 9}$ & 0.7819882 & 0.390994136468 & 1.001599936220 & 0.000470558180 & 0.000556338261 & 85.545532405578 \\
\hline
\end{tabular}


Table 2b. Sun-Mars L2 halo orbit initial conditions and time period at $A 2=0.000005$.

\begin{tabular}{|c|c|c|c|c|c|c|}
\hline $\mathbf{q}$ & $\mathbf{T p}$ & $\mathbf{T p} / 2$ & $\mathbf{x}$ & $\mathbf{z}$ & $\dot{\mathbf{y}}$ & Tp in Days \\
\hline 1 & 3.08791515 & 1.543957579633 & 1.004291664675 & 0.000438068670 & 0.002464489308 & 337.802183824053 \\
\hline 0.99 & 2.46702265 & 1.233511328746 & 1.003829171684 & 0.000446118644 & 0.001798844141 & 269.879707913536 \\
\hline 0.98 & 2.04796398 & 1.023981994188 & 1.003504951398 & 0.000453602351 & 0.001383699753 & 224.036824843153 \\
\hline 0.97 & 1.75678775 & 0.878393876718 & 1.003258131742 & 0.000459029405 & 0.001127519445 & 192.183628441167 \\
\hline 0.96 & 1.54491640 & 0.772458202324 & 1.003062443736 & 0.000462796305 & 0.000963854481 & 169.005982482880 \\
\hline 0.95 & 1.38422796 & 0.692113981915 & 1.002903061991 & 0.000465443977 & 0.000854685513 & 151.427485852213 \\
\hline 0.94 & 1.25814273 & 0.629071367741 & 1.002770423433 & 0.000467357242 & 0.000778917502 & 137.634404343333 \\
\hline 0.93 & 1.15644646 & 0.578223230113 & 1.002658020272 & 0.000468782207 & 0.000724507873 & 126.509350027908 \\
\hline 0.92 & 1.07255922 & 0.536279611695 & 1.002561283407 & 0.000469874420 & 0.000684291103 & 117.332513769047 \\
\hline 0.91 & 1.00206942 & 0.501034710055 & 1.002476923052 & 0.000470733691 & 0.000653826170 & 109.621288473707 \\
\hline 0.9 & 0.94191481 & 0.470957405618 & 1.002402516606 & 0.000471425569 & 0.000630257864 & 103.040680783192 \\
\hline
\end{tabular}

Table 2c. Sun-Mars L2 halo orbit initial conditions and time period at $A 2=0.000010$.

\begin{tabular}{|c|c|c|c|c|c|c|}
\hline $\mathbf{q}$ & $\mathbf{T p}$ & $\mathbf{T p} / 2$ & $\mathbf{x}$ & $\mathbf{z}$ & $\dot{\mathbf{y}}$ & Tp in Days \\
\hline 1 & 3.08810316 & 1.544051581568 & 1.004532284888 & 0.000438151324 & 0.002593923633 & 337.822750489559 \\
\hline 0.99 & 2.49938438 & 1.249692191122 & 1.004111836537 & 0.000445761845 & 0.001941702833 & 273.419915701006 \\
\hline 0.98 & 2.09774420 & 1.048872103231 & 1.003813644389 & 0.000452939289 & 0.001520353500 & 229.482527044489 \\
\hline 0.97 & 1.81486951 & 0.907434758363 & 1.003582609003 & 0.000458309807 & 0.001249941385 & 198.537477387064 \\
\hline 0.96 & 1.60654080 & 0.803270400140 & 1.003396018529 & 0.000462152035 & 0.001070822868 & 175.747377355471 \\
\hline 0.95 & 1.44693774 & 0.723468870941 & 1.003241474745 & 0.000464922015 & 0.000947445638 & 158.287616030613 \\
\hline 0.94 & 1.32065528 & 0.660327640850 & 1.003110988432 & 0.000466964254 & 0.000859336678 & 144.472958364315 \\
\hline 0.93 & 1.21809175 & 0.609045878106 & 1.002999041475 & 0.000468508598 & 0.000794430395 & 133.253031292728 \\
\hline 0.92 & 1.13299615 & 0.566498076887 & 1.002901685370 & 0.000469705351 & 0.000745344509 & 123.944005994176 \\
\hline 0.91 & $\begin{array}{c}1.06113791 \\
6\end{array}$ & 0.530568958076 & 1.002816023536 & 0.000470653774 & 0.000707386916 & 116.083080954845 \\
\hline 0.9 & 0.99955615 & 0.499778076616 & 1.002739886155 & 0.000471420704 & 0.000677470095 & 109.346349883710 \\
\hline
\end{tabular}


Table 2d. Sun-Mars L2 halo orbit initial conditions and time period at $A 2=0.000015$.

\begin{tabular}{ccccccc}
\hline $\mathbf{q}$ & $\mathbf{T p}$ & $\mathbf{T p} / \mathbf{2}$ & $\mathbf{x}$ & $\mathbf{z}$ & $\dot{\mathbf{y}}$ & $\mathbf{T p}$ in Days \\
\hline $\mathbf{1}$ & 3.08816614 & 1.544083071254 & 1.004721683493 & 0.000438206196 & 0.002696109077 & 337.829640112051 \\
$\mathbf{0 . 9 9}$ & 2.52163008 & 1.260815040109 & 1.004324814765 & 0.000445507097 & 0.002050574125 & 275.853481705377 \\
$\mathbf{0 . 9 8}$ & 2.13134432 & 1.065672163060 & 1.004040066922 & 0.000452466871 & 0.001623368973 & 233.158208924383 \\
$\mathbf{0 . 9 7}$ & 1.85364844 & 0.926824221105 & 1.003816611798 & 0.000457783589 & 0.001342282230 & 202.779694235503 \\
$\mathbf{0 . 9 6}$ & 1.64739058 & 0.823695294457 & 1.003633906892 & 0.000461661449 & 0.001151961635 & 180.216136080242 \\
$\mathbf{0 . 9 5}$ & 1.48829289 & 0.744146445053 & 1.003480934547 & 0.000464501967 & 0.001018371111 & 162.811658519558 \\
$\mathbf{0 . 9 4}$ & 1.36171691 & 0.680858456026 & 1.003350586912 & 0.000466622837 & 0.000921389839 & 148.964891493477 \\
$\mathbf{0 . 9 3}$ & 1.25845375 & 0.629226878288 & 1.003237897242 & 0.000468242429 & 0.000848905524 & 137.668428466087 \\
$\mathbf{0 . 9 2}$ & 1.17246024 & 0.586230120376 & 1.003139260759 & 0.000469506801 & 0.000793375878 & 128.261176018571 \\
$\mathbf{0 . 9 1}$ & 1.09961856 & 0.549809283323 & 1.003051996717 & 0.000470514193 & 0.000749933946 & 120.292668039140 \\
$\mathbf{0 . 9}$ & 1.03702991 & 0.518514958162 & 1.002974072281 & 0.000471331779 & 0.000715333612 & 113.445788617082 \\
\hline
\end{tabular}

